\title{
Biomarker resolution of uterine smooth muscle tumor necrosis as benign vs malignant
}

\author{
Eric J Yang and George L Mutter \\ Department of Pathology, Division of Women's and Perinatal Pathology, Harvard Medical School, \\ Brigham and Women's Hospital, Boston, MA, USA
}

\begin{abstract}
Uterine leiomyosarcomas are rare malignant tumors with a poor prognosis while leiomyomas are common benign tumors unrelated to their malignant counterparts. Diagnostic features commonly present in leiomyosarcoma include cytologic atypia, high mitotic index, and a sarcoma-specific geographic cell death designated 'tumor cell necrosis (TCN)'. TCN has a sharp viable-nonviable boundary lacking inflammation, fibrosis or granulation tissue seen in nonspecific infarction. These characteristics are sometimes difficult to interpret on routine hematoxylin and eosin slides, and can lead to diagnostic errors. In this study, we used extracellular matrix stains to test the hypothesis that the host response which characterizes nonspecific infarction may degrade the matrix in infarcted tumor more than in TCN. A 'honeycomb' pattern of reticulin highlighted individual tumor cells in viable regions of all cases. Nonviable area of reticulin patterns differed significantly by diagnosis $(P<0.001)$, with a honeycomb pattern maintained $(91 \%, 20 / 22)$ in leiomyosarcoma and lost $(61 \%, 11 / 18)$ in leiomyomas. Retention of honeycomb reticulin in nonviable areas of leiomyosarcoma occurred irrespective of the presence of inflammation, hemorrhage, fibrosis, or diffuse hyalinization. Fibrosis/ hyalinization as evidenced by trichrome stain was significantly $(P<0.001)$ more common in nonviable areas of benign leiomyomas $(100 \%, 18 / 18)$ compared with leiomyosarcomas $(36 \%, 8 / 22)$. In those occasions where viable tissues contained discernable polarization of mitotic activity, these decreased toward the nonviable interface in leiomyosarcoma, and had an opposite pattern in leiomyomas, increasing toward the interface. There is a significant difference in the reticulin and collagen networks of nonviable areas of leiomyosarcoma compared with leiomyoma. At the time of early injury, both retain reticulin; however, this is cleared over time in benign, but not malignant, areas of necrosis. We conclude that proliferative repair of leiomyomas at the viable-nonviable interface includes remodeling of the extracellular matrix, in contrast to the static preservation of extracellular matrix ('mummification') in nonviable areas of leiomyosarcomas.
\end{abstract}

Modern Pathology (2015) 28, 830-835; doi:10.1038/modpathol.2015.35; published online 20 February 2015

Uterine leiomyosarcomas are rare malignant tumors with a poor prognosis of 28- to 45-month overall survival irrespective of stage. ${ }^{1}$ Conversely, leiomyomas are common benign uterine tumors without clinically significant elevation of risk of malignancy. For decades, diagnosis of malignant smooth muscle tumors centered primarily upon the presence of cytologic atypia and increased mitotic activity ( $>10$ mitoses/10 HPFs). ${ }^{2}$ In 1994 an additional diagnostic feature specific to leiomyosarcoma was introduced: a peculiar form of coagulative tumor cell necrosis

Correspondence: Professor GL Mutter, MD, Department of Pathology, Division of Women's and Perinatal Pathology, Harvard Medical School, Brigham and Women's Hospital, 75 Francis Street, Boston, MA 02115, USA.

E-mail: gmutter@partners.org

Received 9 October 2014; revised 19 December 2014; accepted 21 December 2014; published online 20 February 2015 designated as 'tumor cell necrosis (TCN)'. ${ }^{3}$ New criteria were required to distinguish TCN from the much more common and nonspecific infarctive processes encountered both in benign and malignant smooth muscle tumors. Histologically, both types of necrosis demonstrate coagulative changes, ${ }^{4}$ but differ in the character of the interface between nonviable and viable tissues. TCN has an abrupt transition with minimal tissue reaction, while infarct type necrosis commonly exhibits inflammation, hemorrhage, and a zone of granulation/fibrous (reparative) tissue that separates the viable and nonviable areas of tumor. ${ }^{5}$ Ghosts of individual necrotic cells are retained more commonly in TCN. Despite these specific criteria, interpretation of nonviable areas as sarcoma-specific vs nonspecific can be difficult and poorly reproducible. A recent study showed that a panel of expert gynecologic pathologists reached full agreement for the presence or absence of TCN in 


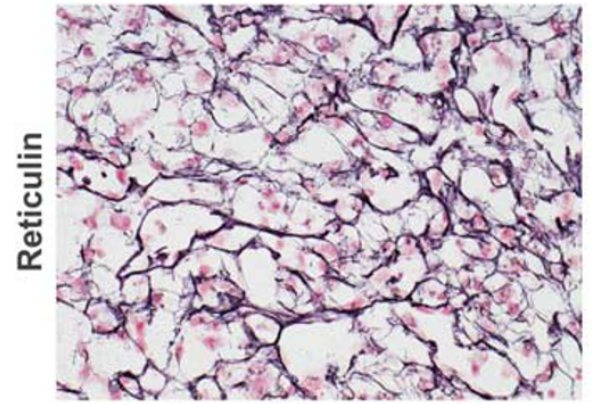

Present (honeycomb)

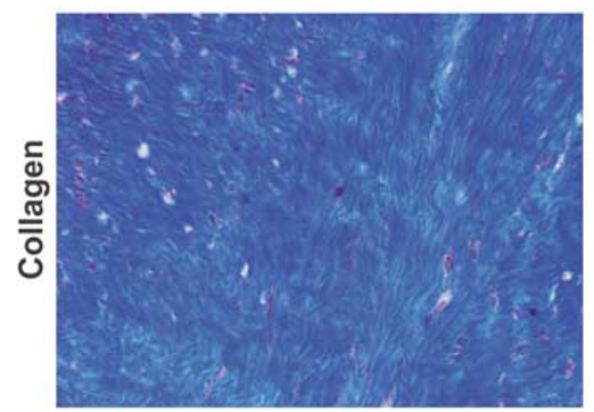

Present (acellular)

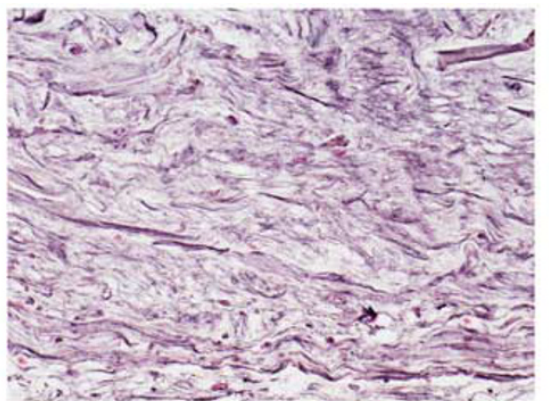

Present (linear)

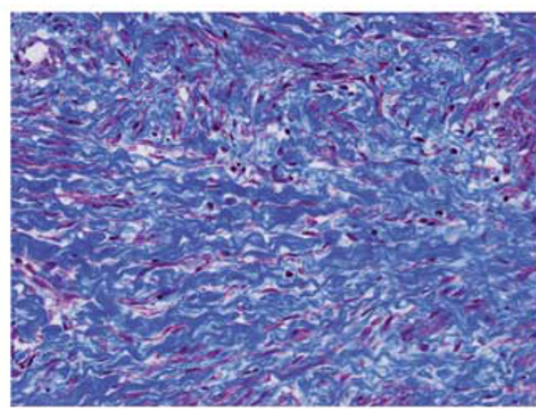

Present (cellular)

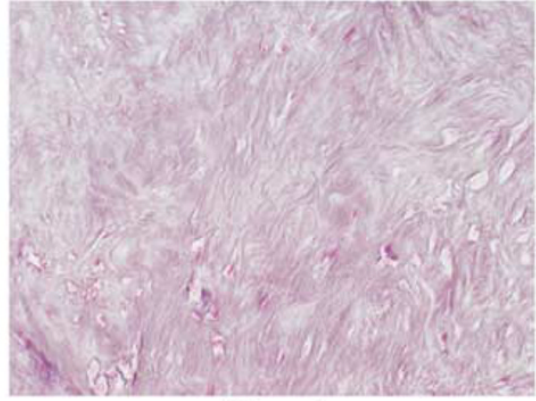

Absent

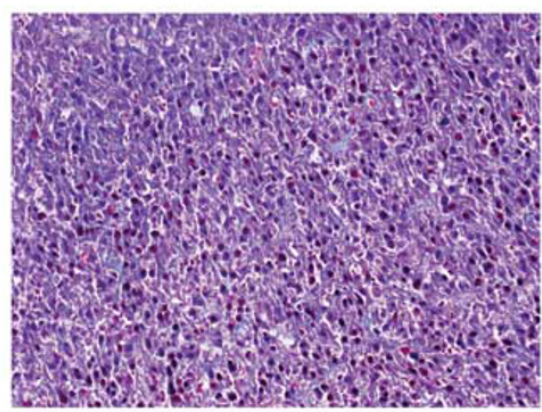

Absent

Figure 1 Scored patterns of reticulin network (reticulin stain, reticulin fibers are black, top row) and collagen (trichrome, collagen is blue, bottom row) deposition within nonviable regions of smooth muscle tumors.

only $35 \%$ of the leiomyosarcoma cases evaluated. ${ }^{6}$ Points of disagreement included intervening fibrocollagenous tissue, hyalinization, hemorrhage, and inflammation. These results suggest that TCN and ischemic-type necrosis exist on a histologic spectrum and may not be readily distinguished by hematoxylin and eosin (H\&E) morphologic criteria alone.

Discriminating histologic criteria for TCN compared with infarct type necrosis are all related to tissue reactions to cell death, and to some extent the duration of coagulative necrosis. We hypothesized that in addition to those informative differences which have been described at the nonviable to viable interface, there may be cumulative differences within the nonviable areas themselves. In this study, we used special stains (reticulin and trichrome) to examine reticulin and collagen extracellular components within nonviable tumor areas in leiomyomas and leiomyosarcomas to test the hypothesis that they are qualitatively different. Additionally, to test the idea that the viable areas also are qualitatively different, we looked for mitotic gradients polarized toward, compared with away from, the nonviable areas.

\section{Materials and methods}

Twenty-two cases of leiomyosarcoma and 18 cases of leiomyoma of the uterus were retrieved from the archives of Brigham and Women's Hospital, using the report diagnosis as an initial screen. Two pathologists (GLM and EJY) reviewed the slides to confirm the diagnosis and to select a representative block with a viable-nonviable tumor interface. Serial sections from the selected blocks were stained for H\&E, reticulin (modified Gordon and Sweet method, Reticulin II staining Kit, Cat\#860-024, Nexus Special Stainer, Ventana-Roche), trichrome (Gomori Trichrome Blue, Poly Scientific, Bay Shore, NY, USA), and Ki67 antigen (MIB1 antibody from DAKO, Carpinteria, CA, USA, Cat.M7240, diluted 1:100). The largest nonviable to viable interface was identified, including areas on both sides of the boundary. For slides from each block, this region was marked on all serial sections to permit coordinated observations within the same tissue region across all stains.

Reticulin patterns within the nonviable areas (Figure 1) were scored based upon the preservation of a darkly staining reticulin network, and its geometric pattern as follows: (1) absent (reticulin degraded); (2) present, honeycomb pattern (preserved polygonal network delimiting individual or groups of cells); and (3) present, linear pattern (network preserved, but distorted by compression to parallel fibers). Blue-staining collagen as visualized by trichrome stain was scored in the nonviable area as present or absent (Figure 1), where presence of collagen was variable associated with an acellular or cellular background. Immunohistochemical staining for mitotic activity (MIB1) was scored within the viable area flanking the viable-nonviable interface (Figure 2). When mitotic activity was present, changing density relative to the interface was scored 


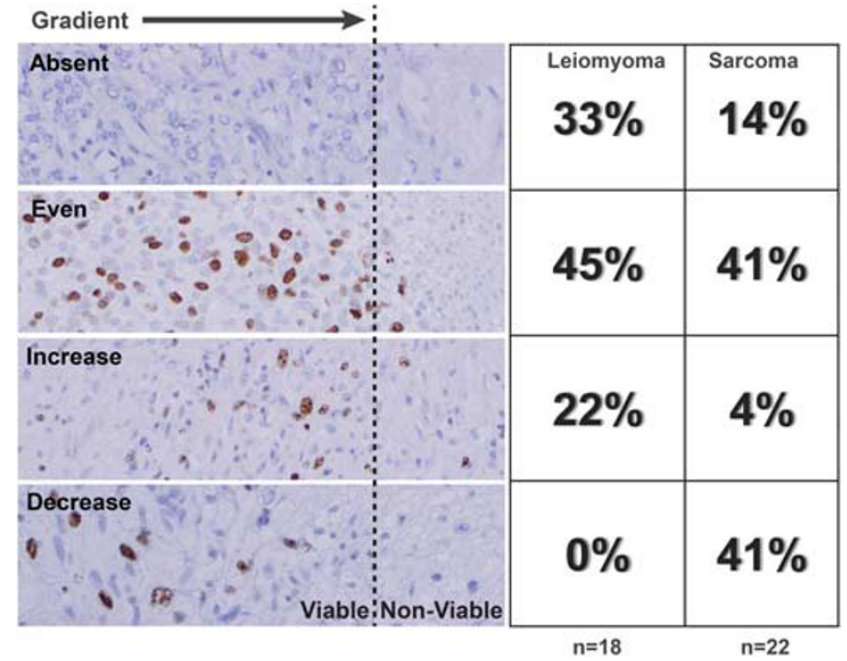

Figure 2 Mitotic patterns (MIB1 stain) within viable areas flanking the viable (left)-nonviable (right) interface (dashed line). In many cases of leiomyoma and leiomyosarcoma, mitotic activity is absent, or evenly distributed in the viable tissues immediately flanking the interface. When a discernable mitotic gradient is present, it tends to increase toward the interface in leiomyomas, and decrease toward the interface in leiomyosarcomas $(P<0.001)$.

as increasing or decreasing toward the interface. $\chi^{2}$-test and Fisher's exact tests were used to determine statistical significance (Systat v13.1, San Jose, CA, USA).

\section{Results}

Representative photomicrographs of staining patterns across the viable to nonviable interface are shown in Figure 3 for all stains. Summary data appears in Table 1.

\section{Reticulin Stain (Fibers $=$ Black)}

In the viable tissue of both leiomyosarcomas and leiomyomas (Figure 3) reticulin highlighted individual tumor cells, creating a 'honeycomb' appearance. There were three reticulin patterns in the nonviable tumor: (1) honeycomb-retention of the native reticulin pattern, (2) absent-loss of any reticulin staining, and (3) linear-reticulin staining in a linear pattern without the native honeycomb pattern. Reticulin pattern in nonviable areas differed significantly by diagnosis (leiomyosarcoma vs leiomyoma; $P<0.001$ ), with the maintenance of a honeycomb pattern predominating $(91 \%, 20 / 22)$ in leiomyosarcomas and reticulin loss most common $(61 \%, 11 / 18)$ in leiomyomas (Figure 3). Reticulin was retained in nonviable leiomyosarcoma regardless of the histological pattern seen at the interface; inflammation, hemorrhage, fibrosis, and diffuse hyalinization were all seen in association with reticulin retention. In contrast, hyalinization was the predominant histologic pattern in nonviable leiomyoma, and this was always associated with complete loss of reticulin staining. When reticulin was retained in leiomyomas $(33 \%, 6 / 18)$, the viable-nonviable interface had features of early injury, with edema, hemorrhage, and inflammation, but without evidence of repair (granulation tissue formation or significant fibrosis/hyalinization). Retention of reticulin in a linear pattern was a minority finding but seen in both groups (1/18 leiomyoma and 1/22 leiomyosarcoma).

\section{Collagen Stain $($ Trichrome, Collagen $=$ Blue $)$}

Trichrome stain highlighted prominent collagen bundles in blue within viable leiomyoma, but collagen was much less prominent in the viable regions of leiomyosarcoma. In the nonviable tumor, the collagen pattern was categorized as either present or absent (Figure 1). Collagen pattern differed significantly within the nonviable regions by diagnosis $(P<0.001)$, with all leiomyomas $(100 \%, 18 / 18)$ demonstrating abundant (blue) collagen while majority of leiomyosarcoma $(64 \%, 14 / 22)$ lacked staining (Figure 3). In leiomyomas, hyalinization and loss of reticulin usually corresponded to the dense sheets of acellular collagen, but occasionally scattered myocytes were retained (Figure 1, collagen present - 'cellular'). Leiomyomata that retained reticulin in nonviable areas (early injury) demonstrated ropey collagen bundles of pattern and abundance similar to that seen in the viable areas, suggesting a process of retention in the nonviable region rather than de novo collagen formation. A minority of leiomyosarcomas $(36 \%, 8 / 22)$ demonstrated fibrosis and hyalinization of nonviable areas, however unlike their benign counterparts all of these tumors retained reticulin staining (Supplementary Figure 1).

\section{Proliferative Activity (MIB1 Immunostain)}

Proliferative activity evaluated by MIB1 immunostaining was scored in viable tumor tissues flanking the viable-nonviable tumor interface (Figure 2). In the majority of leiomyomas and leiomyosarcomas, MIB1 staining was either absent (leiomyoma 33\% and leiomyosarcoma 14\%); or present evenly in the viable tumor immediately adjacent to the nonviable tumor (leiomyoma 45\%, leiomyosarcoma 41\%). However, in a minority of cases, a MIB1 gradient was present relative to the interface. Mitotic density generally increased toward the interface in leiomyomas $(22 \%)$, and decreased toward the interface in leiomyosarcomas (44\%). The pattern/quantity of proliferative activity adjacent to the nonviable tumor was specifically evaluated in this study. Mitotic activity in regions distant from the interface was not scored and noted to be present even when quiescent at the interface. 

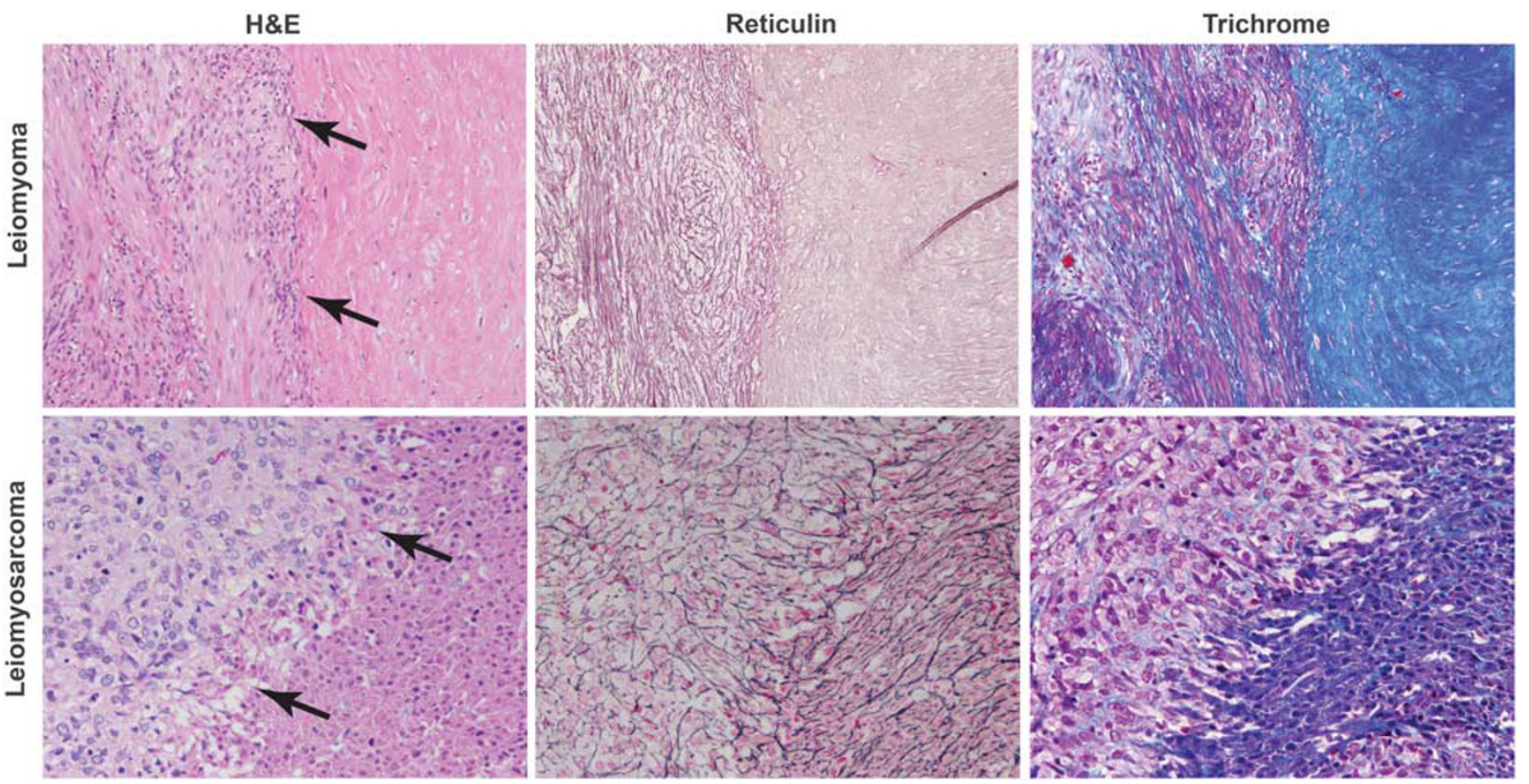

Figure 3 Representative examples of viable to nonviable interfaces (arrows, viable left, nonviable right) in leiomyoma (upper row) and leiomyosarcoma (bottom row) stained by hematoxylin and eosin (H\&E; left), reticulin (center) and trichrome (right). Note retention of honeycomb reticulin network and absence of (blue) collagen in the nonviable areas of the leiomyosarcoma, but not in the leiomyoma. Nonviable areas were significantly more likely to retain a reticulin network within leiomyosarcomas (96\%) than leiomyomas (40\%), as summarized in Table 1. Collagen deposition in nonviable areas showed the opposite, being present in only $36 \%$ of leiomyosarcomas compared to $100 \%$ of leiomyomas.

Table 1 Biomarker patterns by diagnosis (benign/malignant)

\begin{tabular}{|c|c|c|c|c|c|}
\hline Marker & Area scored & Staining pattern & $\begin{array}{c}\text { Benign } \\
\text { (leiomyoma) }\end{array}$ & $\begin{array}{c}\text { Malignant } \\
\text { (leiomyosarcoma) }\end{array}$ & Significance \\
\hline \multirow[t]{3}{*}{ Reticulin } & \multirow[t]{3}{*}{ Nonviable } & Absent & $61.1 \%$ & $4.5 \%$ & \multirow[t]{3}{*}{$P<0.001 \chi^{2}$} \\
\hline & & Present-honeycomb & $33.3 \%$ & $90.9 \%$ & \\
\hline & & Present-linear & $5.6 \%$ & $4.5 \%$ & \\
\hline \multirow[t]{2}{*}{ Collagen } & \multirow{2}{*}{ Nonviable } & Absent & $0 \%$ & $63.6 \%$ & \multirow{2}{*}{$P<0.001$ Fisher exact } \\
\hline & & Present & $100 \%$ & $36.4 \%$ & \\
\hline \multirow[t]{5}{*}{ Mitotic activity (MIB1) } & \multirow[t]{5}{*}{$\begin{array}{l}\text { Viable area, gradient } \\
\text { toward interface }\end{array}$} & Absent & $33.3 \%$ & $13.6 \%$ & \multirow[t]{5}{*}{$P=0.009 \chi^{2}$} \\
\hline & & Even & $44.4 \%$ & $40.9 \%$ & \\
\hline & & Increase & $22.2 \%$ & $4.5 \%$ & \\
\hline & & Decrease & $0 \%$ & $40.9 \%$ & \\
\hline & & Total $(100 \%)$ & 18 & 22 & \\
\hline
\end{tabular}

\section{Discussion}

Our study examined nonviable ('necrotic') regions of benign and malignant uterine smooth muscle tumors, including those in which the viable-nonviable interface had a variety of histologic features: some with no tissue reaction, and others with inflammation, hemorrhage, fibrosis, and/or hyalinization. Most strikingly, we found that the reticulin network is retained (95\%) in the nonviable regions of leiomyosarcoma regardless of the histologic appearance of the tumor at the interface (Figure 3, Table 1).
In contrast, nonviable areas within leiomyomas most commonly $(61 \%)$ completely lost the reticulin network. In less frequent cases (39\%) of leiomyomas retaining reticulin in nonviable regions, features of early injury were present. This suggests that clearance of reticulin within leiomyomas occurs over time as part of the host response, but this process is lacking in the nonviable areas of leiomyosarcomas. Trichrome stain showed collagen deposition, in a pattern of (acellular) hyalinization or (cellular) fibrosis, within nonviable regions of a subset of leiomyomas and leiomyosarcomas. In leiomyomas, 
collagen deposition was always associated with reticulin loss; however, in leiomyosarcomas, reticulin was retained despite overlying hyalinization or fibrosis. Proliferative activity in viable tissues, as evaluated by MIB1, was sometimes polarized as a gradient toward the interface. This differed between benign and malignant tumors, as mitotic density tended to increase toward the interface in leiomyomas, and decrease toward the interface in leiomyosarcomas. We interpret this increase of mitotic activity seen at the interface of leiomyomas as a regenerative, or repair type process which is most active in immediate proximity to the nonviable zone. Conversely, suppression of mitotic activity toward the necrotic interface of leiomyosarcomas implies an ongoing degenerative process lacking a substantial host response.

The mechanism of cell death in benign and malignant smooth muscle tumors may be different. Both leiomyomas and leiomyosarcomas experience severe hypoxia; however, leiomyomas do not show evidence of hypoxic stress while leiomyosarcomas demonstrate strong induction of hypoxic stress markers (HIF- $1 \alpha / \mathrm{HIF} 2-\alpha$, GLUT-1 and CA IX). ${ }^{7-9}$ This implies that leiomyomas are well-adapted to low oxygen levels and therefore, a precipitous hypoxic event (eg, acute vascular compromise by torsion or compression and iatrogenic vessel occlusion by embolization beads) may be the principal cause of infarctive changes. Indeed, the histologic appearance of hyalinized nonviable leiomyoma is identical to that of an embolized leiomyoma. ${ }^{10}$ Infarcted leiomyoma tend to be accompanied by devitalized vessels, ${ }^{6}$ further supporting an acute vascular event causing localized ischemia. Analogous to strokes and myocardial infarctions, the affected areas undergo cell death via multiple mechanisms, including a central area of necrosis and a surrounding mantle of ischemic tissue that undergo apoptosis. ${ }^{4,11-13}$ The robust inflammatory response to the necrotic core initiates tissue degradation and stromal matrix remodeling, resulting in the replacement of infarcted tumor by dense hyalinized tissue. ${ }^{5}$

The nonviable regions of leiomyosarcoma tend to display preservation of preexisting vessels with a peripheral rim of viable neoplastic cells, ${ }^{6}$ implying that ischemic injury in leiomyosarcoma is not primarily of vascular etiology, but rather a more complex process acting upon viable cells adjacent to the outer perimeter of necrosis. In contrast to leiomyomas, leiomyosarcoma overexpress markers of hypoxic stress, ${ }^{9}$ in keeping with a chronic and ongoing state of injurious ischemia rather than the abrupt vascular events that characterize infarctions in leiomyomas. Depending on the duration/severity of ischemia and consequent ATP depletion, cells may undergo either necrosis (with abrupt/complete ATP depletion) or apoptosis (with ATP retention above a threshold). ${ }^{11,14}$ Given the apparent chronicity of leiomyosarcoma ischemia, we conjecture a

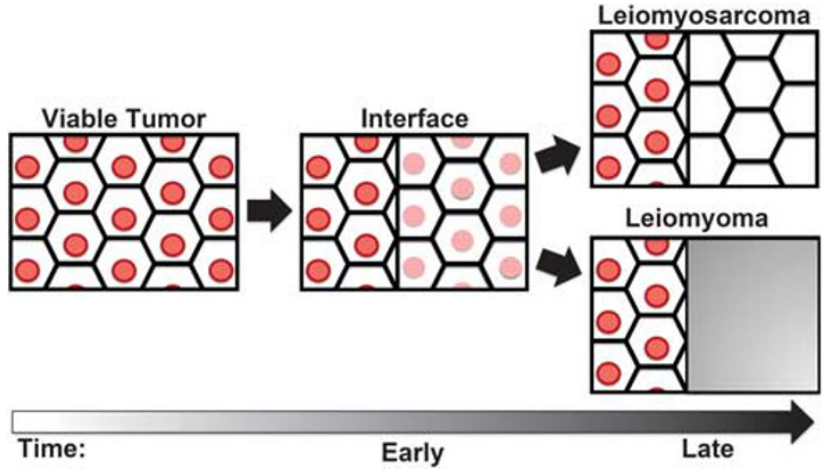

Figure 4 Model of nonspecific (infarction) compared with malignant tumor cell necrosis (TCN; leiomyosarcoma) over time. In both processes there is myocyte dropout in the nonviable zone, but the interface with viable tissue remains relatively stable (static) in infarction, compared with the dynamic repositioning seen over time in TCN. In infarction the reticulin network is cleared to be replaced by mature collagen. This contrasts with malignant TCN, where the 'mummified' nonviable zone retains its reticulin and lacks significant new collagen deposition.

predominance of apoptotic cell death over necrosis. This is in keeping with the relative lack of inflammatory tissue response to cell death and associated retention of the native reticulin network in leiomyosarcoma. ${ }^{3,5,6}$

These findings can be summarized in a model (Figure 4) in which reticulin is retained in the early phases of necrosis of both leiomyomas and leiomyosarcomas. In leiomyomas the necrosis develops as a discrete event, producing a stable viable-nonviable interface that is subjected to host repair over time, including matrix remodeling, collagen deposition, and reticulin resorption. In contrast, the necrosis of leiomyosarcomas is progressive and dynamic over time, producing a migratory interface that expands outwards over time, preventing development of a regenerative host response at any fixed location.

Therefore, we propose that for both leiomyoma and leiomyosarcoma, ischemia is the predominant inciting source of injury, followed by cell death via necrosis and/or apoptosis depending on the duration and magnitude of the ischemic insult. The relative abundance of necrosis vs apoptosis determines the associated tissue response; necrosis promotes inflammation, hemorrhage, and fibrosis, and apoptosis results in an inert interface. This is in keeping with the concept and histologic description of TCN and infarct type necrosis, and places the two types of 'necrosis' on a histologic and mechanistic spectrum rather than being discrete entities.

In conclusion, we have found that nonviable areas of leiomyosarcoma retain the native 'honeycomb' reticulin network while nonviable leiomyoma loses reticulin over time. We speculate that this difference in the stromal matrix within the nonviable regions is codetermined by the extent of dynamic mobility of the viable-nonviable interface over time, in addition to differing tissue responses to alternate mechanisms 
of cell death. Our findings are consistent with the current diagnostic distinction between leiomyosarcoma TCN and leiomyoma infarct type necrosis, and provide insight as to why these entities can be difficult to distinguish. Given that nonviable regions of both leiomyosarcoma and leiomyoma can retain reticulin staining, the diagnostic utility of special stains for reticulin are limited. However, as nearly all nonviable areas of leiomyosarcoma retain reticulin, the loss of reticulin is more compatible with a benign than a malignant process. Finally, studies of leiomyosarcoma and smooth muscle tumors of uncertain malignant potential suggest that the isolated presence of TCN is not necessarily predictive of aggressive behavior. ${ }^{15-18}$ Although the retention of reticulin in nonviable tissues of a uterine smooth muscle tumor can be indicative of a malignant lesion, the significance of this finding should always be interpreted within the context of cytologic atypia and mitotic activity.

\section{Disclosure/conflict of interest}

The authors declare no conflict of interest.

\section{References}

1 Lim D, Wang WL, Lee CH, et al. Old versus new FIGO staging systems in predicting overall survival in patients with uterine leiomyosarcoma: a study of 86 cases. Gynecol Oncol 2013;128:322-326.

2 Silverberg SG, Kurman RJ. Tumors of the Uterine Corpus and Gestational Trophoblastic Disease. Fascicle 3. 3rd series. Armed Forces Institute of Pathology: Washington, DC, USA, 1991; pp 130-139.

3 Bell SW, Kempson RL, Hendrickson MR. Problematic uterine smooth muscle neoplasms. A clinicopathologic study of 213 cases. Am J Surg Pathol 1994;18:535-558.

4 Majno G, Joris I. Apoptosis, oncosis, and necrosis. An overview of cell death. Am J Pathol 1995;146:3-15.

5 Kempson RL, Hendrickson MR. Smooth muscle, endometrial stromal, and mixed Müllerian tumors of the uterus. Mod Pathol 2000;13:328-342.
6 Lim D, Alvarez T, Nucci MR, et al. Interobserver variability in the interpretation of tumor cell necrosis in uterine leiomyosarcoma. Am J Surg Pathol 2013;37: 650-658.

7 Younes M, Lechago LV, Somoano JR, Mosharaf M, Lechago J. Wide expression of the human erythrocyte glucose transporter Glut1 in human cancers. Cancer Res 1996;56:1164-1167.

8 Zhong H, De Marzo AM, Laughner E, et al. Overexpression of hypoxia-inducible factor 1alpha in common human cancers and their metastases. Cancer Res 1999;59:5830-5835.

9 Mayer A, Höckel M, Wree A, et al. Lack of hypoxic response in uterine leiomyomas despite severe tissue hypoxia. Cancer Res 2008;68:4719-4726.

10 Colgan TJ, Pron G, Mocarski EJM, et al. Pathologic features of uteri and leiomyomas following uterine artery embolization for leiomyomas. Am J Surg Pathol 2003;27:167-177.

11 Sendoel A, Hengartner MO. Apoptotic cell death under hypoxia. Physiology (Bethesda) 2014;29:168-176.

12 Krijnen PAJ, Nijmeijer R, Meijer CJLM, et al. Apoptosis in myocardial ischaemia and infarction. J Clin Pathol 2002;55:801-811.

13 Burke AP, Virmani R. Pathophysiology of acute myocardial infarction. Med Clin North Am 2007;91: 553-572.

14 Lieberthal W, Menza SA, Levine JS, Graded ATP. depletion can cause necrosis or apoptosis of cultured mouse proximal tubular cells. Am J Physiol 1998;274: F315-F327.

15 Ip PPC, Cheung ANY, Clement PB. Uterine smooth muscle tumors of uncertain malignant potential (STUMP): a clinicopathologic analysis of 16 cases. Am J Surg Pathol 2009;33:992-1005.

16 Peters WA3rd, Howard DR, Andersen WA, Figge DC. Uterine smooth-muscle tumors of uncertain malignant potential. Obstet Gynecol 1994;83: 1015-1020.

17 Perrone T, Dehner LP. Prognostically favorable 'mitotically active' smooth-muscle tumors of the uterus. A clinicopathologic study of ten cases. Am J Surg Pathol 1988;12:1-8.

18 Atkins KA, Arronte N, Darus CJ, Rice LW. The use of p16 in enhancing the histologic classification of uterine smooth muscle tumors. Am J Surg Pathol 2008;32: 98-102.

Supplementary Information accompanies the paper on Modern Pathology website (http://www.nature.com/ modpathol) 